\title{
Siedlungsbedingter Finanzbedarf von Kommunen
}

\section{Siedlungsstruktur messen und im Kommunalen Finanzausgleich berücksichtigen}

\author{
Ulrich Keilmann/Marc Gnädinger/Heike Weyell
}

Die weitgehend exogen bestimmten siedlungstypischen Eigenschaften einzelner Städte und Gemeinden beeinflussen deren Finanzbedarf. Einschlägig sind die Anzahl der Ortsteile, die Fläche, die Einwohnerdichte, die Aufteilung der Fläche u.v.a.m. Diese spontan einleuchtende These beruhte bislang weitgehend auf anekdotischer Evidenz: Es ist bisher nicht gelungen, den Einfluss der Siedlungsstruktur auf die konkreten kommunalen Finanzbedarfe wissenschaftlich valide zu bestimmen. In Hessen wurde dazu nunmehr mit wissenschaftlicher Unterstützung ein Siedlungsindex entwickelt. Ausgehend von dieser Messgröße kann mit einheitlichen Geobasisdaten die unterschiedliche Siedlungsstruktur (von zentriert bis zersiedelt) definiert und sich daraus ergebende Mehrbedarfe einzelner Kommunen errechnet werden.

Die Überörtliche Prüfung kommunaler Körperschaften (ÜPKK) in Hessen hat mit ihrer 203. Vergleichenden Prüfung ${ }^{1}$ untersucht, wie das mehrdimensionale

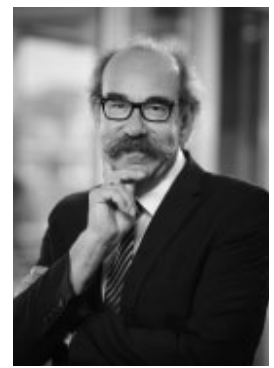

Dr. Ulrich Keilmann

Leiter der Abteilung Überörtliche Prüfung kommunaler

Körperschaften in Hessen

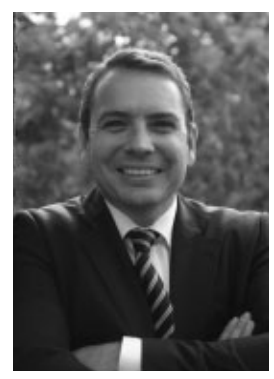

Dr. Marc Gnädinger

Leiter Grundsatzreferat der Überörtlichen Prüfung kommunaler Körperschaften in Hessen
Phänomen der Siedlungsstruktur in einem Siedlungsindex zusammengefasst werden kann. In die Prüfung waren insgesamt 40 Gemeinden mit Einwohnerzahlen von 2.557 bis 17.261 Einwohnern einbezogen. Der Index wurde sodann auch für alle 423 hessischen Städte und Gemeinden errechnet. Mit der Prüfung wurden zusätzlich Vor- und Nachteile, die sich aus der Struktur der zu versorgenden Gemeindefläche ergeben, identifiziert und analysiert. Die Prüfung wurde von Prof. Dr. Lenk (Kompetenzzentrum Öffentliche Wirtschaft, Infrastruktur und Daseinsvorsorge e.V.) wissenschaftlich begleitet.

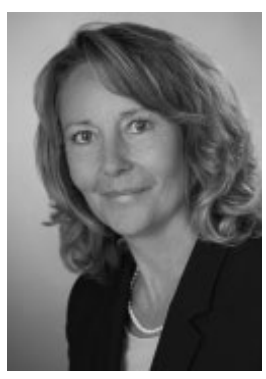

Heike Weyell

Referentin im Prüfungsreferat der Überörtlichen Prüfung kommunaler Körperschaften in Hessen.

\section{Siedlungsindex}

Siedlungsstruktur lässt sich nicht über eine einzelne Kennzahl messen. Für den durch die Überörtliche Prüfung in Hessen entwickelten Siedlungsindex wurden daher verschiedene Indikatoren in einem Gesamtmaß zusammengefasst. Jeder integrierte Indikator misst dabei verschiedene Aspekte der Siedlungsstruktur. ${ }^{2}$ Der Siedlungsindex gibt als Messgröße das Ausmaß der Zersiedlung einer einzelnen Gemeinde wieder - in ihm werden drei Indikatoren zusammengefasst:

- Der Indikator Grad der Streuung der Siedlungen misst die Verteilung der Gemeindeteile im Gemeindegebiet. Gemessen wird die Dispersion durch den Nächster-Nachbar-Index.

- Der Grad der urbanen Durchdringung misst als weiterer Indikator den Anteil der Siedlungs- und Verkehrsfläche an der Gesamtfläche der Gemeinde.

- Die Ausnutzungsdichte misst mittels der Einwohner- und Arbeitsplatzdichte, wie intensiv die Siedlungsfläche einer Gemeinde genutzt wird.

Diese drei Indikatoren gehen für die Berechnung gewichtet in das Gesamtmaß für den Siedlungsindex ein. Je größer der errechnete Siedlungsindex ist, desto größer ist die Zersiedlung.

Vgl. ÜPKK (2018), S. $78 \mathrm{ff}$.

2 Zu Möglichkeiten der Erfassung von Siedlungsstrukturen vgl. exemplarisch Jaeger et al. (2015), S. $15 \mathrm{ff}$. 
Basierend auf der Empfehlung des Kompetenzzentrums Öffentliche Wirtschaft, Infrastruktur und Daseinsvorsorge e.V. unterteilte die Überörtliche Prüfung die Kommunen für eine anschauliche Darstellung anhand ihres Indexwertes in vier Cluster. Zentriert (C1), eher zentriert (C2), eher zersiedelt (C3) und zersiedelt (C4).

\section{Siedlungsrelevanz}

In Bezug auf den Gesamthaushalt zeigte sich zunächst kein statistisch nachweisbarer Zusammenhang zwischen Siedlungsindex und Jahresergebnissen der geprüften Kommunen. Das war darauf zurückzuführen, dass der Gesamthaushalt durch eine Vielzahl von Einflussfaktoren bestimmt wurde. So gilt zunächst die Pflicht zum Haushaltsausgleich ( $\mathbb{9} 92$ Absatz 4 HGO) für alle Kommunen, ob zersiedelt oder zentriert gleichermaßen. Die Siedlungsstruktur war nur ein denkbarer $\mathrm{Pa}$ rameter, so dass sich bei einer Gesamtbetrachtung kein Zusammenhang zwischen der Siedlungsstruktur und dem Ergebnis des Gesamthaushalts nachweisen ließ. Dieses Ergebnis korrespondierte mit Feststellungen des Gutachtens zum Kommunalen Finanzausgleich in Hessen aus dem Jahr 2012. ${ }^{3}$

Erst der erweiterte Aufgabenblick ermöglichte die Sicht auf die speziellen Belastungen zersiedelter Gemeinden. Ausgehend von den berechneten Siedlungsindizes wurde der Einfluss der Siedlungsstruktur auf alle wesentlichen Aufgabenbereiche der geprüften Gemeinden mittels Regressionsanalysen analysiert. Untersucht wurde, ob die Fehlbeträge der einzelnen Aufgaben von der Höhe des Siedlungsindex abhängig waren. Tatsächlich konnte nur in wenigen Aufgabenbereichen ein statistischer signifikanter und kausaler Zusammenhang festgestellt werden: Bei der Feuerwehr, bei den Gemeindestraßen und bei den freiwilligen Bürgerhäusern. In allen drei Bereichen zeigte sich eine statistische Signifikanz. Die Defizite steigen pro Kopf mit zunehmendem Zersiedlungsgrad an.

Für die Beurteilung, ob zwischen dem Anstieg der Fehlbeträge im Bereich der Feuerwehr und dem steigenden Siedlungsindex eine Kausalität vorlag, analysier-

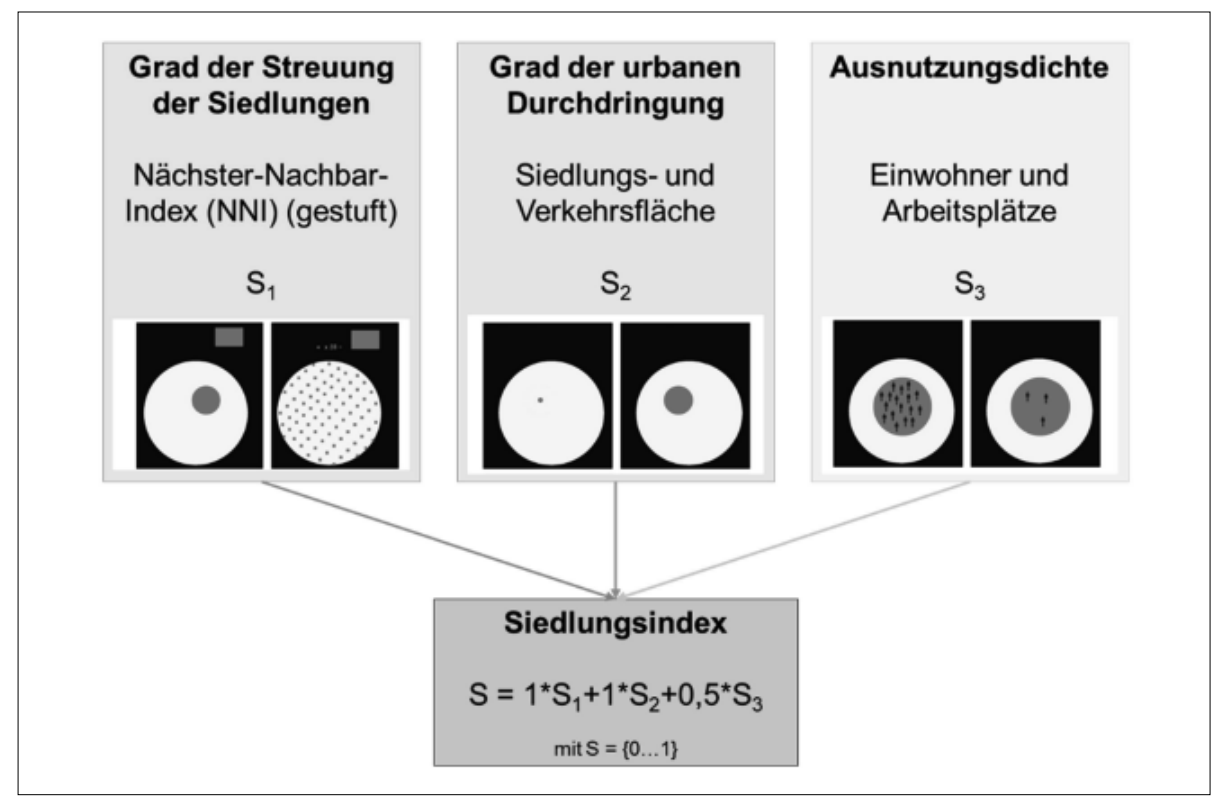

Abb. 1: Siedlungsindex der Überörtlichen Prüfung (Quelle: Kompetenzzentrum Öffentliche Wirtschaft, Infrastruktur und Daseinsvorsorge e.V. mit Grafiken aus Jaeger et al. 2015)

te die Überörtliche Prüfung die Zahl der Feuerwehrgerätehäuser je 1.000 Einwohner in Abhängigkeit zum Siedlungsindex. Dabei zeigte sich ein starker linearer $\mathrm{Zu}-$ sammenhang zwischen Siedlungsindex und Zahl der Feuerwehrgerätehäuser je 1.000 Einwohner. Zentrierte Gemeinden (C1) hatten im Durchschnitt 0,17 Feuerwehrgerätehäuser je 1.000 Einwohner. Zersiedelte Gemeinden (C4) hatten durchschnittlich 2,56 Feuerwehrgerätehäuser je 1.000 Einwohner. Somit hatten zersiedelte Gemeinden durchschnittlich das 15-fache an Feuerwehrgerätehäusern im Vergleich zu wenig zersiedelten Gemeinden.

Ein ähnlicher Befund zeigte sich bei den Straßen. Zum Aufgabenbereich Straßen zählten der Bau, die Instandhaltung, Beleuchtung und Entwässerung von Gemeindestraßen. Hier zeigte sich ebenfalls, dass die Fehlbeträge mit dem Grad der Zersiedlung steigen. Für die Beurteilung, ob zwischen dem Anstieg der Fehlbeträge und einem steigenden Siedlungsindex eine Kausalität vorlag, wurde für dieses Feld eine Analyse der Straßeninfrastruktur vorgenommen. Hierzu wurde die Länge der Gemeindestraßen in km je 1.000 Einwohner in Abhängigkeit zum Siedlungsindex untersucht. Dabei zeigte sich ein starker linearer Zusammenhang von Straßenlänge und Siedlungsindex. Zentrierte Gemeinden (C1) hatten im Durchschnitt Straßenlängen von 4,18 km je 1.000 Einwohner. Zersiedelte Gemeinden (C4) hatten hingegen durchschnittlich $8,40 \mathrm{~km}$ Straßen je
1.000 Einwohner. Somit hatten zersiedelte Gemeinden durchschnittlich mehr als das doppelte an km Straßen je 1.000 Einwohner im Vergleich zu zentrierten Gemeinden.

Bei den Bürgerhäusern gab die Regressionsgerade ebenfalls Hinweise auf einen Zusammenhang von Zersiedlung und den Fehlbeträgen je Einwohner. Bei der Plausibilisierung zeigte sich ein linearer Zusammenhang zwischen der Zahl der Bürgerhäuser je 1.000 Einwohner und dem Siedlungsindex. Zentrierte Gemeinden (C1) hatten im Durchschnitt 0,22 Bürgerhäuser je 1.000 Einwohner. Zersiedelte Gemeinden (C4) hatten durchschnittlich 2,64 Bürgerhäuser je 1.000 Einwohner. Somit hatten zersiedelte Gemeinden durchschnittlich das 12-fache an Bürgerhäusern im Vergleich zu zentrierten Gemeinden.

\section{Sonderfall Ver- und Entsorgung}

Bei den Gebührenhaushalten Wasser und Abwasser stellte sich die Situation anders da. Hier zeigte die Regressionsgerade beispielsweise im Bereich Abwasser keine statistisch signifikante Steigung. Damit lag die Vermutung nahe, dass die Siedlungsstruktur keinen Einfluss auf die Wirtschaftlichkeit der Aufgabenerfüllung hatte. Dies war allerdings darauf zurückzuführen, dass für den Gebührenhaushalt

3 Vgl. Grüttner/Hesse/Lenk (2012). 
Abwasser das Kostendeckungsprinzip des $\mathbb{1} 10$ KAG gilt. Das Kostendeckungsprinzip kann dazu führen, dass der Bürger verpflichtet wird, hohe Gebühren zu entrichten, wenn die spezifischen Entsorgungskosten hoch sind. Dies kann zu einer Benachteiligung der Bürger in zersiedelten Gemeinden führen - zumindest dann, wenn sie höhere Gebühren zu entrichten haben als Bürger zentrierter Gemeinden.

Zur Analyse, ob die Bürger in Gemeinden mit einem hohen Siedlungsindex mehr belastet waren, hat die Überörtliche Prüfung daher für den Gebührenhaushalt Abwasser fiktive Gebühren je Gemeinde ermittelt. Diese fiktive Gebühr war die Gebühr, die für einen Ausgleich des
Jahr zu Jahr ändern können, ist der Siedlungsindex regelmäßig neu zu berechnen. Das ist insbesondere dann ratsam, wenn eintreten, Gebietsreformen durchgeführt werden $^{4}$ und insbesondere, wenn vom Siedlungsindex finanzielle Förderungen (vor allem im kommunalen Finanzausgleich - KFA) abhängig gemacht werden.

Hessen hat einen bedarfsorientierten Kommunalen Finanzausgleich, ist mithin vom Verbundquotenmodell abgekehrt. ${ }^{5}$ Bei der dem hessischen Finanzausgleichgesetz (FAG HE) zugrunde liegenden Bedarfsorientierung ist die Berücksichtigung der Zersiedlung als Sonderbedarf zu prüfen. Von dieser Empfehlung ist nur dann abzusehen, wenn das FAG HE bereits demographiebedingt starke Änderungen

Evaluation des Kommunalen Finanzausgleichs $\mathrm{zu}$ berücksichtigen. ${ }^{6}$ Inhaltlich wird u.a. zu überlegen sein, wie genau der Siedlungsindex in die Definition des „ländlichen Raums“ zu integrieren ist. Das könnte primär Aufgabe der Expertenkommission, die sich mit der Zukunft der „Zentralen Orte“ und der Raumstruktur in Hessen auseinandersetzt (ZORa) sein.

\section{Literatur}

CDU Hessen/BÜNDNISgo/DIE GRÜNEN Hessen (2018): Koalitionsvertrag zwischen CDU Hessen und BÜNDNISgo/DIE GRÜNEN Hessen für die 20. Legislaturperiode, unter https:// www.gruene-hessen.de/partei/files/2018/12/ Koalitionsvertrag-CDU-GR\%C $3 \% 9$ CNE-2018Stand-20-12-2018-online.pdf [Zugriff am 12.02.2019].

Grüttner, A./Hesse, M./Lenk, T. (2012): Finanzwissenschaftliches Gutachten zur Fortschreibung des Kommunalen Finanzausgleichs in Hessen, Flächenansatz und Demografieansatz, Soziallasten, Leipzig.

Hessisches Ministerium der Finanzen (o.J.): Neuordnung des Kommunalen Finanzausgleichs ab dem Jahr 2016, unter https://finanzen.hessen.de/sites/default/files/media/hmdf/ kfa_2016_-_gesamtpraesentation_nach_verabschiedung_des_gesetzes.pdf [Zugriff am 08.03.2019]

Jaeger, J./Schwick, C./Hennig, I.E./Orlitova, E./Soukup, T./Nazarnia, N./Kienast, F., (2015): Zersiedelung aus landschaftsökologischer, sozialer und siedlungstechnischer Sicht; in: WSL Berichte - Forum für Wissen, 2015, Heft 33, S. 15-25, unter https://www.dora.lib4ri.ch/wsl/islandora/object/wsl\%3A9062/datastream/PDF/ view [Zugriff am 08.03.2019]

Stadt Oberzent (o.J.): Grenzänderungsvertrag der Stadt Beerfelden und der Gemeinden Hesseneck, Rothenberg und Sensbachtal, Vertragstext zum 2. Mai 2017, Abruf unter https://www.stadt-oberzent.de/stadtleben/ stadtportrait/fusion/grenzaenderungsvertragoriginal-2.mai-2017.pdf?cid=be [Zugriff am 06.03.2019]

Überörtliche Prüfung kommunaler Körperschaften - Präsident des Hessischen Rechnungshofs (2018): Kommunalbericht 2018, zugleich Landtag, Drucksache 19 / 6812 dex künftighin verbessert werden. Aktuell erhalten nicht alle als zersiedelt geltenden Gemeinden eine Förderung als ländlicher Raum und umgekehrt. gleiche Ergebnis. Auch hier werden die Bürger zersiedelter Kommunen stärker belastet.

\section{Siedlungsstruktur und Kommunaler Finanzausgleich}

Die Berechnung des Siedlungsindex erfolgte im Jahr 2017. Da die gemeindeindividuellen Werte der Indikatoren sich von
Die Chancen für eine Optimierung der Förderung des ländlichen Raums im KFA stehen gut: Im Koalitionsvertrag zwischen CDU und Bündnis 90/Die Grünen in Hessen wurden die Prüfergebnisse der Überörtlichen Prüfung benannt. Dort ist in Aussicht gestellt, die Erkenntnisse aus der Prüfung bei der aktuell anstehenden
4 Dazu zählen auch freiwillige Gemeindefusionen. In Hessen z.B. die Fusion von vier Gemeinden zur Stadt Oberzent zum 1. Januar 2018. Vgl. Stadt Oberzent (o.J.).

5 Zum bedarfsorientierten KFA in Hessen vgl. exemplarisches Hessisches Ministerium der Finanzen (o.J.).

6 Vgl. CDU Hessen/BÜNDNIS9o/DIE GRÜNEN Hessen (2018), S. 171. 